\title{
Size-Dependent Brittle-to-Ductile Transition in Silica Glass Nanofibers
}

Junhang Luo ${ }^{1, \#}$, Jiangwei Wang ${ }^{1, \#}$, Erik Bitzek ${ }^{2,3, \#}$, Jian Yu Huang ${ }^{4}$, He Zheng ${ }^{1}$, Limin Tong, ${ }^{5}$, Qing Yang ${ }^{5}, \mathrm{Ju} \mathrm{Li}^{2}$,, Scott X. Mao ${ }^{1, *}$

${ }^{1}$ Department of Mechanical Engineering \& Materials Science, University of Pittsburgh, Pittsburgh, Pennsylvania 15261, USA

${ }^{2}$ Department of Nuclear Science and Engineering and Department of Materials Science and Engineering, Massachusetts Institute of Technology, 77 Massachusetts Avenue, Cambridge, Massachusetts 02139, USA

${ }^{3}$ Department of Materials Science and Engineering, Institute I, Friedrich-Alexander-Universität Erlangen-Nürnberg (FAU), 91058 Erlangen, Germany

${ }^{4} 8915$ Hampton Ave NE, Albuquerque, New Mexico 87122, USA

${ }^{5}$ State Key Laboratory of Silicon Materials, Zhejiang University, Hangzhou 310027, China

\section{Supplementary Information}

1. Additional results of the tensile tests

2. Additional molecular dynamics simulation results

3. Images about sample preparation 


\section{Additional results of the tensile tests}

The beam was blanked during the tensile tests to exclude the irradiation on the deformation behavior of $\mathrm{SiO}_{2}$ nanofiber. The morphologies of pristine and fractured nanofibers were captured to reveal the fracture mode. Supporting Figure S1 shows the deformation and loading-unloading response of a $\mathrm{SiO}_{2}$ nanofiber with the diameter of $18.7 \mathrm{~nm}$. The nanofiber was firstly deformed via loading-unloading test and then pulled again until fracture. The $\mathrm{SiO}_{2}$ nanofiber of $18.7 \mathrm{~nm}$ shows a typical brittle fracture, as demonstrated by the flat fracture surface (Supporting Figure S1c) and the stress-strain curve (Supporting Figure S1e). The fracture strength of $18.7 \mathrm{~nm} \mathrm{SiO}_{2}$ nanofiber was about 3.7 GPa, with a Young's modulus of $77.6 \mathrm{GPa}$ (Supporting Figure S1d-e). Before the sudden fracture, the maximum elastic strain occurred in the $18.7 \mathrm{~nm}$ nanofiber was $4.8 \%$, which is over 1.5 times that of the $33.9 \mathrm{~nm}$ diameter nanofiber (Figure 1f in the main text). The increase in strength and elastic strain limit can be attributed to the size-dependent strengthening.

Supporting Figure S2 show an example about the ductile fracture of a $4.7 \mathrm{~nm}$ nanofiber with $18 \%$ tensile elongation. The nanofiber is deformed under the strain rate of $2.8 \times 10^{-4} \mathrm{~s}^{-1}$. Significant diameter shrunk and localized necking is observed in the fracture morphologies, confirming the ductile fracture mode. Supporting Figure S3 shows the fracture morphologies of $\mathrm{SiO}_{2}$ nanofibers under higher strain rates. The fracture of $22.4 \mathrm{~nm}$ under a high strain rate of $4.4 \times 10^{-2} \mathrm{~s}^{-1}$ shows a shear fracture feature (Supporting Figure S3a), while a localized necking is observed in $2.8 \mathrm{~nm}$ nanofiber under the strain rate of $4.7 \times 10^{-2} \mathrm{~s}^{-1}$, similar to the fracture behaviors under low strain rate (Figure $1 \mathrm{~g}-\mathrm{h}$ in the main text).

To reveal the effects of electron beam irradiation on the deformation, a $\mathrm{SiO}_{2}$ nanofiber with the diameter up to $128.8 \mathrm{~nm}$ were deformed under the strain rate of $2.7 \times 10^{-4} \mathrm{~s}^{-1}$. A superplastic deformation was observed before the fracture of this 128.8 $\mathrm{nm} \mathrm{SiO}{ }_{2}$ nanofiber (Supporting Figure S4), in contrast to the brittle behavior of 33.9 $\mathrm{nm}$ and $18.7 \mathrm{~nm}$ nanofibers when deformed under beam-blank conditions (Figure 1b-c in the main text and Supporting Figure S1), even though the diameters are much smaller in the beam-blank deformation cases. The control experiments suggest that the beam irradiation has negligible influences on the deformation.

Moreover, in our experiments, 72 individual $\mathrm{SiO}_{2}$ nanofibers with different diameters were fabricated and tested under various deformation strain rate at room temperature. Their diameters, deformation conditions, fracture strengths and plastic elongations are summarized in Supporting Table S1 and plotted in Figure 2 in the main text. The engineering fracture strength was calculated by $4 \Delta \mathrm{F} / \pi \mathrm{d}_{0}{ }^{2}$, where $\Delta \mathrm{F}$ refers to the force at the fracture point and $\mathrm{d}_{0}$ is the initial diameter of the nanofiber. The plastic elongation was calculated by $\left(\mathrm{L}_{\mathrm{f}}-\mathrm{L}_{0}\right) / \mathrm{L}_{0}$, where $\mathrm{L}_{0}$ and $\mathrm{L}_{\mathrm{f}}$ are the initial and final (i.e. determined after fracture) gauge length of the nanofiber. 

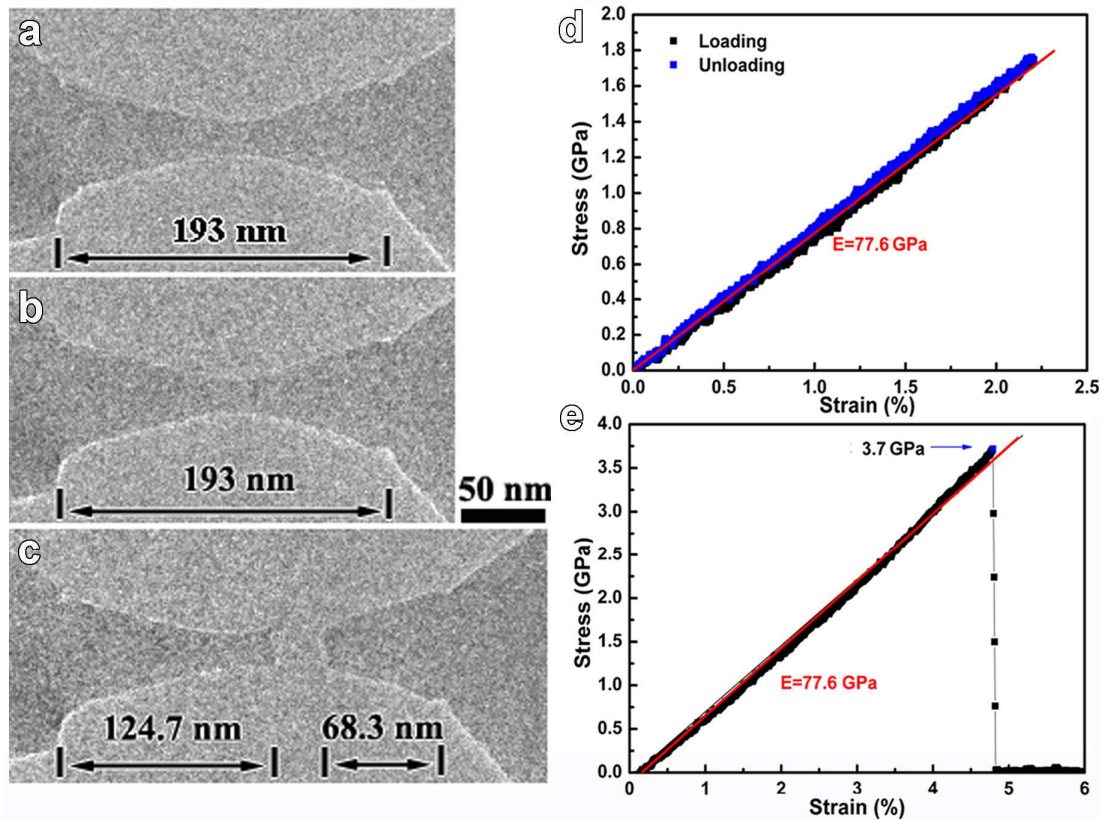

Figure S1 Mechanical tests on the $\mathrm{SiO}_{2}$ nanofiber with the diameter of $18.7 \mathrm{~nm}$. a, b and $\mathbf{c}$ are images of the nanofiber before, after cycle loading and final fracture; $\mathbf{d}$ and e are the strain-stress curves for the cycle loading and tensile test.

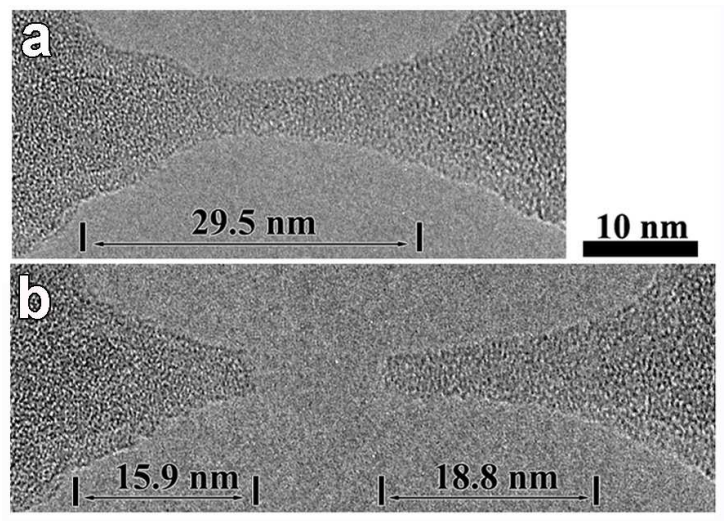

Figure S2 Ductile fracture of a $4.7 \mathrm{~nm} \mathrm{SiO}_{2}$ nanofiber with an elongation of $\sim 18 \%$. a, the pristine nanofiber and $\mathbf{b}$, the fractured nanofiber after tensile deformation at a strain rate of $2.8 \times 10^{-4} \mathrm{~s}^{-1}$.
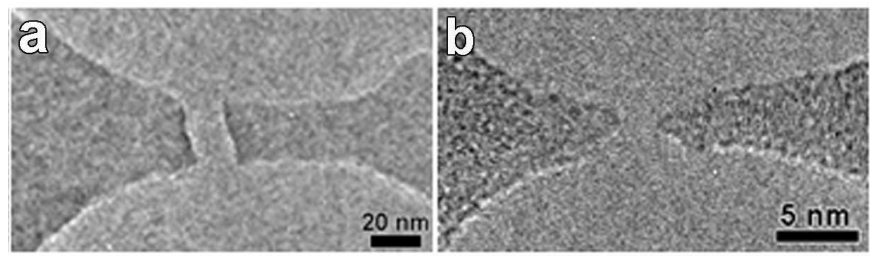

Figure S3 Fracture morphologies of $\mathrm{SiO}_{2}$ nanofibers under higher strain rates. a, a shear fracture of a $22.4 \mathrm{~nm}$ nanofiber at a strain rate of $4.4 \times 10^{-2} \mathrm{~s}^{-1} ; \mathbf{b}$, localized necking of a $2.8 \mathrm{~nm}$ nanofiber at a strain rate of $4.7 \times 10^{-2} \mathrm{~s}^{-1}$. 


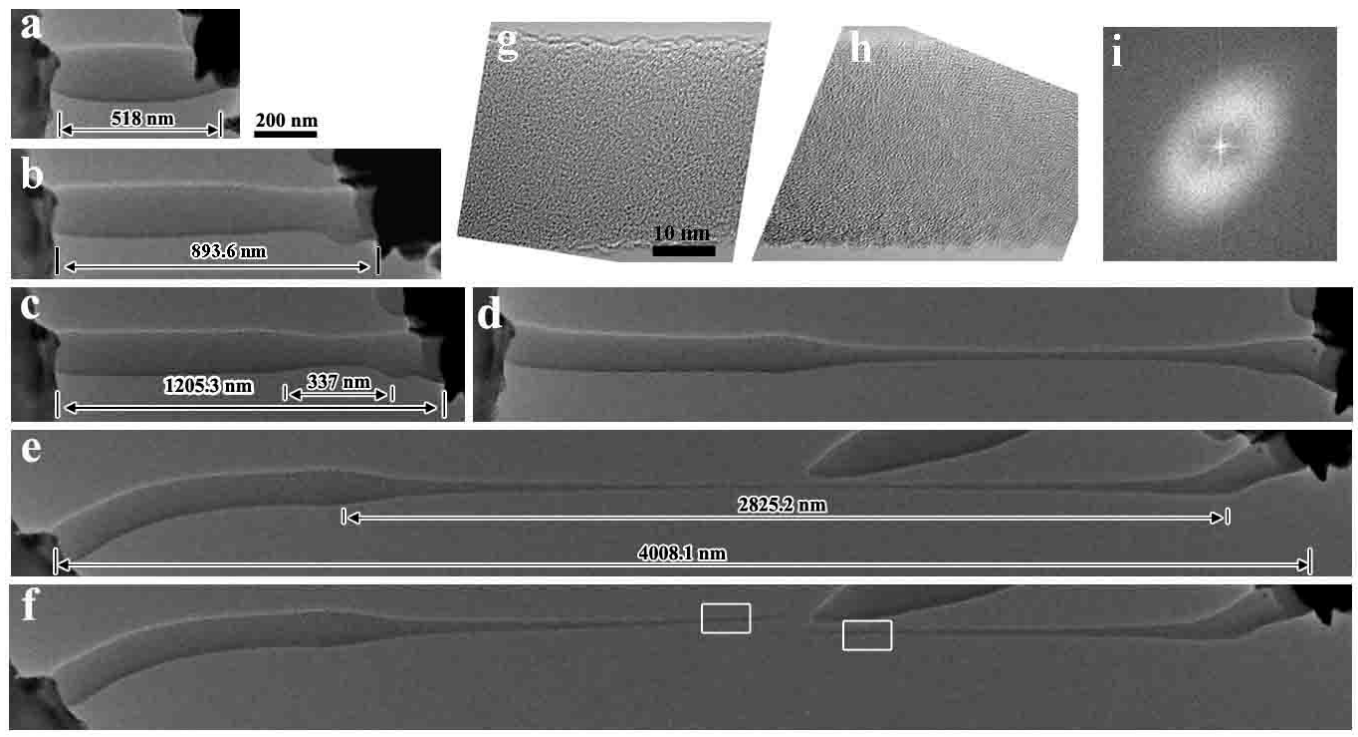

Figure S4. In situ tensile elongation of a $128.8 \mathrm{~nm} \mathrm{SiO}{ }_{2}$ nanofiber at strain rate of $2.7 \times 10^{-4} \mathrm{~s}^{-1}$. (a) $\mathrm{t}=0 \mathrm{~min}$, tensile test beginning. (b) $\mathrm{t}=22 \mathrm{~min}$, local thinning happened at the place near the contacting area of the STM probe side. (c) $t=69$ min, a dog-bond-shaped sample; (d) $\mathrm{t}=212 \mathrm{~min}$, the dog-bond-shaped sample uniformly elongated along the wire axis; (e) $\mathrm{t}=246 \mathrm{~min}$, the nanowire just before failure; (f) Failure of the nanowire. $(\mathrm{g})$ and $(\mathrm{h})$ are HRTEM images of the white framed regions in (f). (i) FFT of (h).

Table S1 Summary of fracture strengths and plastic elongations of $\mathrm{SiO}_{2}$ nanofibers

\begin{tabular}{|c|c|c|c|c|}
\hline Sample & $\begin{array}{c}\text { Initial } \\
\text { diameter } \\
(\mathrm{nm}) \\
d_{0}\end{array}$ & $\begin{array}{c}\text { Elongation } \\
(\%) \\
\left(L_{f}-L_{0}\right) / L_{0}\end{array}$ & $\begin{array}{c}\text { Fracture } \\
\text { stress } \\
(\mathrm{GPa}) \\
4 \Delta F / d_{0}{ }^{2}\end{array}$ & $\begin{array}{c}\text { Strain rate } \\
\left(\mathrm{s}^{-1}\right)\end{array}$ \\
\hline NW1-1 & 1.6 & 3.4 & 10.0 & $6.1 \times 10^{-2}$ \\
\hline NW1-2 & 3.8 & 3.4 & 9.2 & $1.2 \times 10^{-1}$ \\
\hline NW1-3 & 4.5 & 1.6 & 8.5 & $1.7 \times 10^{-1}$ \\
\hline NW1-4 & 5.7 & 1.3 & 5.8 & $1.6 \times 10^{-1}$ \\
\hline NW1-5 & 8.4 & 0.5 & 4.9 & $5.0 \times 10^{-1}$ \\
\hline NW1-6 & 10.8 & 0.9 & 4.5 & $1.1 \times 10^{-1}$ \\
\hline NW1-7 & 13.9 & 0.5 & 2.1 & $1.3 \times 10^{-1}$ \\
\hline NW1-8 & 17.2 & 0.6 & 2.0 & $7.1 \times 10^{-1}$ \\
\hline NW1-9 & 18.2 & 0.6 & 4.3 & $1.2 \times 10^{-1}$ \\
\hline NW1-10 & 18.6 & 0.0 & 1.9 & $6.0 \times 10^{-2}$ \\
\hline NW1-11 & 18.7 & 0.0 & 3.7 & $8.4 \times 10^{-2}$ \\
\hline NW1-12 & 22.0 & 0.0 & 4.8 & $6.2 \times 10^{-2}$ \\
\hline NW1-13 & 27.0 & 0.0 & 2.9 & $6.6 \times 10^{-2}$ \\
\hline NW1-14 & 33.5 & 0.0 & 1.7 & $5.2 \times 10^{-2}$ \\
\hline NW1-15 & 35.4 & 0.0 & 1.6 & $5.8 \times 10^{-2}$ \\
\hline NW1-16 & 40.0 & 0.0 & 1.3 & $1.8 \times 10^{-2}$ \\
\hline NW1-17 & 45.0 & 0.0 & 1.6 & $4.7 \times 10^{-2}$ \\
\hline NW2-1 & 1.1 & - & 13.2 & 7.2 \\
\hline NW2-2 & 2.8 & 14.6 & & \\
\hline
\end{tabular}




\begin{tabular}{|c|c|c|c|c|}
\hline NW2-3 & 3.7 & 13.1 & 4.8 & $9.4 \times 10^{-3}$ \\
\hline NW2-4 & 4.7 & 10.5 & 6.3 & $4.2 \times 10^{-2}$ \\
\hline NW2-5 & 5.3 & 8.2 & 3.9 & $1.1 \times 10^{-2}$ \\
\hline NW2-6 & 6.3 & 8.6 & 4.9 & $6.9 \times 10^{-3}$ \\
\hline NW2-7 & 6.6 & 6.2 & 2.3 & $2.3 \times 10^{-2}$ \\
\hline NW2-8 & 7.1 & 4.6 & 9.1 & $1.8 \times 10^{-2}$ \\
\hline NW2-9 & 8.1 & 3.9 & 3.5 & $2.1 \times 10^{-2}$ \\
\hline NW2-10 & 9.9 & 1.3 & 4.2 & $2.8 \times 10^{-2}$ \\
\hline NW2-11 & 10.8 & 1.0 & 4.6 & $1.1 \times 10^{-2}$ \\
\hline NW2-12 & 10.8 & 0.7 & 6.0 & $4.8 \times 10^{-2}$ \\
\hline NW2-13 & 11.0 & 1.0 & 5.2 & $4.3 \times 10^{-2}$ \\
\hline NW2-14 & 11.6 & 1.4 & 7.4 & $1.6 \times 10^{-2}$ \\
\hline NW2-15 & 12.5 & 1.2 & 2.4 & $4.3 \times 10^{-2}$ \\
\hline NW2-16 & 14.5 & 1.1 & 3.1 & $8.1 \times 10^{-3}$ \\
\hline NW2-17 & 15.9 & 0.6 & 5.4 & $4.9 \times 10^{-2}$ \\
\hline NW2-18 & 16.8 & 0.8 & 5.3 & $4.2 \times 10^{-2}$ \\
\hline NW2-19 & 17.7 & 0.6 & 4.8 & $3.9 \times 10^{-2}$ \\
\hline NW2-20 & 19.1 & 0.0 & 3.9 & $4.6 \times 10^{-2}$ \\
\hline NW2-21 & 19.6 & 0.0 & 3.1 & $2.7 \times 10^{-2}$ \\
\hline NW2-22 & 21.5 & 0.0 & 4.9 & $4.8 \times 10^{-2}$ \\
\hline NW2-23 & 22.4 & 0.0 & 4.2 & $4.4 \times 10^{-2}$ \\
\hline NW2-24 & 33.9 & 0.0 & 2.2 & $3.3 \times 10^{-2}$ \\
\hline NW2-25 & 40.0 & 0.0 & 1.9 & $3.3 \times 10^{-2}$ \\
\hline NW2-26 & 44.8 & 0.0 & 0.8 & $4.2 \times 10^{-2}$ \\
\hline NW2-27 & 47.6 & 0.0 & 1.0 & $4.8 \times 10^{-2}$ \\
\hline NW3-1 & 2.8 & - & 7.5 & $2.0 \times 10^{-3}$ \\
\hline NW3-2 & 3.9 & 11.8 & 4.7 & $1.8 \times 10^{-3}$ \\
\hline NW3-3 & 3.9 & 16.7 & N/A & $6.2 \times 10^{-4}$ \\
\hline NW3-4 & 5.6 & 9.05 & 4.3 & $4.0 \times 10^{-3}$ \\
\hline NW3-5 & 5.6 & 14.5 & 4.1 & $7.9 \times 10^{-4}$ \\
\hline NW3-6 & 8.5 & 5.4 & 4.4 & $3.6 \times 10^{-3}$ \\
\hline NW3-7 & 8.6 & 3.8 & 4.4 & $2.4 \times 10^{-3}$ \\
\hline NW3-8 & 12.1 & 4.5 & - & $5.2 \times 10^{-4}$ \\
\hline NW3-9 & 13.3 & 2.5 & 3.3 & $6.4 \times 10^{-4}$ \\
\hline NW3-10 & 19.8 & 0.0 & 1.2 & $1.3 \times 10^{-3}$ \\
\hline NW3-11 & 35.1 & 0.0 & 2.5 & $2.5 \times 10^{-3}$ \\
\hline NW4-1 & 4.4 & 15.1 & - & $4.8 \times 10^{-4}$ \\
\hline NW4-2 & 4.6 & 16.5 & - & $3.3 \times 10^{-4}$ \\
\hline NW4-3 & 4.7 & 17.6 & - & $2.8 \times 10^{-4}$ \\
\hline NW4-4 & 5.0 & 14.1 & - & $2.0 \times 10^{-4}$ \\
\hline NW4-5 & 5.1 & 15.1 & 5.3 & $4.5 \times 10^{-4}$ \\
\hline NW4-6 & 5.5 & 15.5 & - & $2.8 \times 10^{-4}$ \\
\hline NW4-7 & 5.8 & 11.5 & 4.6 & $4.4 \times 10^{-4}$ \\
\hline NW4-8 & 5.9 & 11.3 & 2.9 & $3.5 \times 10^{-4}$ \\
\hline NW4-9 & 6.0 & 13.3 & - & $1.8 \times 10^{-4}$ \\
\hline NW4-10 & 6.0 & 11.7 & - & $3.4 \times 10^{-4}$ \\
\hline NW4-11 & 7.0 & 11.1 & 2.3 & $3.5 \times 10^{-4}$ \\
\hline NW4-12 & 7.2 & 9.4 & - & $3.7 \times 10^{-4}$ \\
\hline
\end{tabular}




\begin{tabular}{|c|c|c|c|c|}
\hline NW4-13 & 7.8 & 8.0 & - & $2.4 \times 10^{-4}$ \\
\hline NW4-14 & 8.1 & 7.8 & 1.7 & $2.7 \times 10^{-4}$ \\
\hline NW4-15 & 9.4 & 6.4 & - & $3.2 \times 10^{-4}$ \\
\hline NW4-16 & 15.1 & 2.3 & - & $1.9 \times 10^{-4}$ \\
\hline NW4-17 & 15.3 & 1.6 & 2.4 & $1.5 \times 10^{-4}$ \\
\hline NW4-18 & 16.6 & 1.6 & - & $2.4 \times 10^{-4}$ \\
\hline NW4-19 & 21.1 & 0.0 & - & $2.5 \times 10^{-4}$ \\
\hline
\end{tabular}

-: Image not clear; Force not recorded

\section{Additional MD simulation results}

Supporting Figure S5 shows the snapshots of the PBC sample under uniaxial tension. Comparing to Fig. 3 of the paper one sees that the fracture surface is orthogonal to the tensile axis, whereas the fracture plane in the wire is inclined to the tensile axis. This is an indication that the failure of the wire has an additional shear component.

It is clear that the failure behavior in the simulation of silica glass depends strongly on the interaction potential. We therefore performed simulations with the three-body potential of Vashishta et al. and the two-body potential by van Best, Kramer and van Santen (BKS). It is important to note that both potentials lead to different defect structures in the unstrained glass samples. In particular, the BKS potential allows for the existence of over-coordinated atoms, which are not observed in the Vashishta samples. As can be seen from the stress-strain curves in Supporting Figure S6 and the snapshots in Supporting Figure S7, with the BKS potential, both the wire and the bulk sample deform in a ductile fashion. It is interesting to note that by suppressing the stress relaxation orthogonal to the tensile axis leads to brittle fracture of the PBC sample. However, these boundary conditions, also frequently used in MD simulations of glass fracture, do not represent the situation in experimental (uniaxial) tensile tests. From the bond switching (S) and loss (L) statistics (Supporting Figure S6) one sees clearly that more atoms are switching bonding partner than losing bonds. The S/L statistics during deformation can be thus used to characterize the ductility or brittleness of a given interaction potential. 


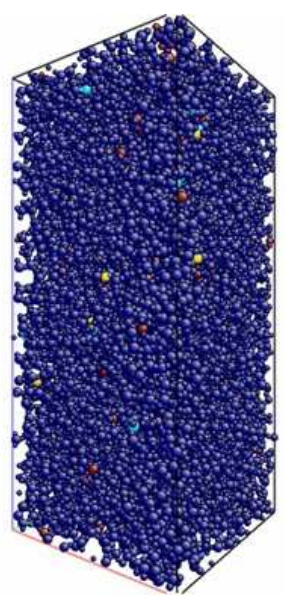

$10 \%$

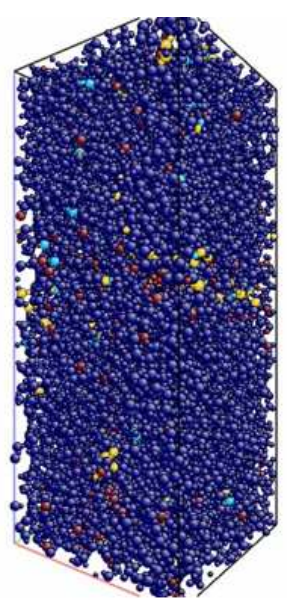

$15 \%$

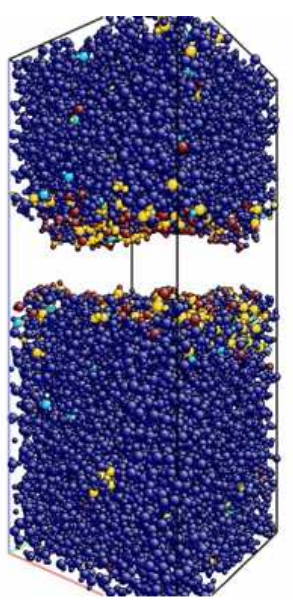

$20 \%$

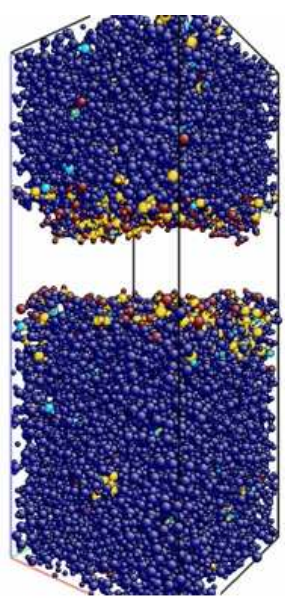

$23 \%$

Figure S5 Snapshots from the bulk Vashishta sample at different strains. The atoms are colored according to the changes of their bonding topology: dark blue atoms have exactly the same neighbors as in the strain free initial configuration, light blue atoms have gained a bond, yellow atoms have switched bonding partners while maintaining the same coordination number, red corresponds to broken bonds.

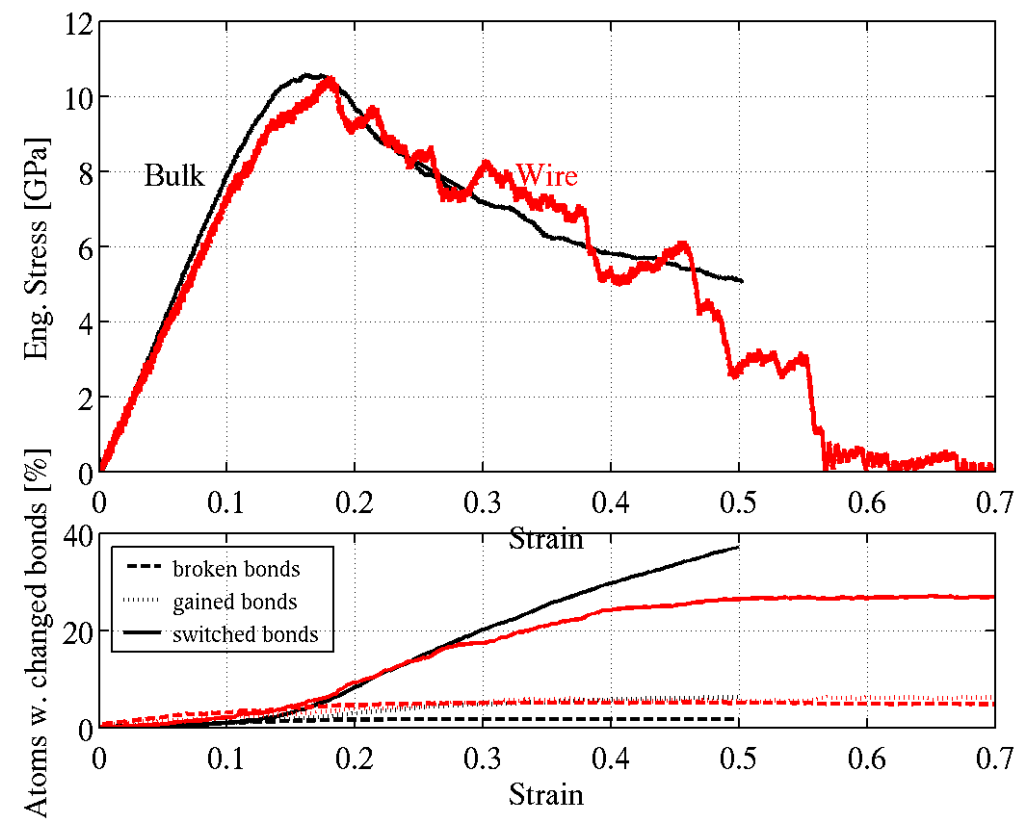

Figure S6 Stress-Strain response for the BKS sample together with the bond-change statistics. 

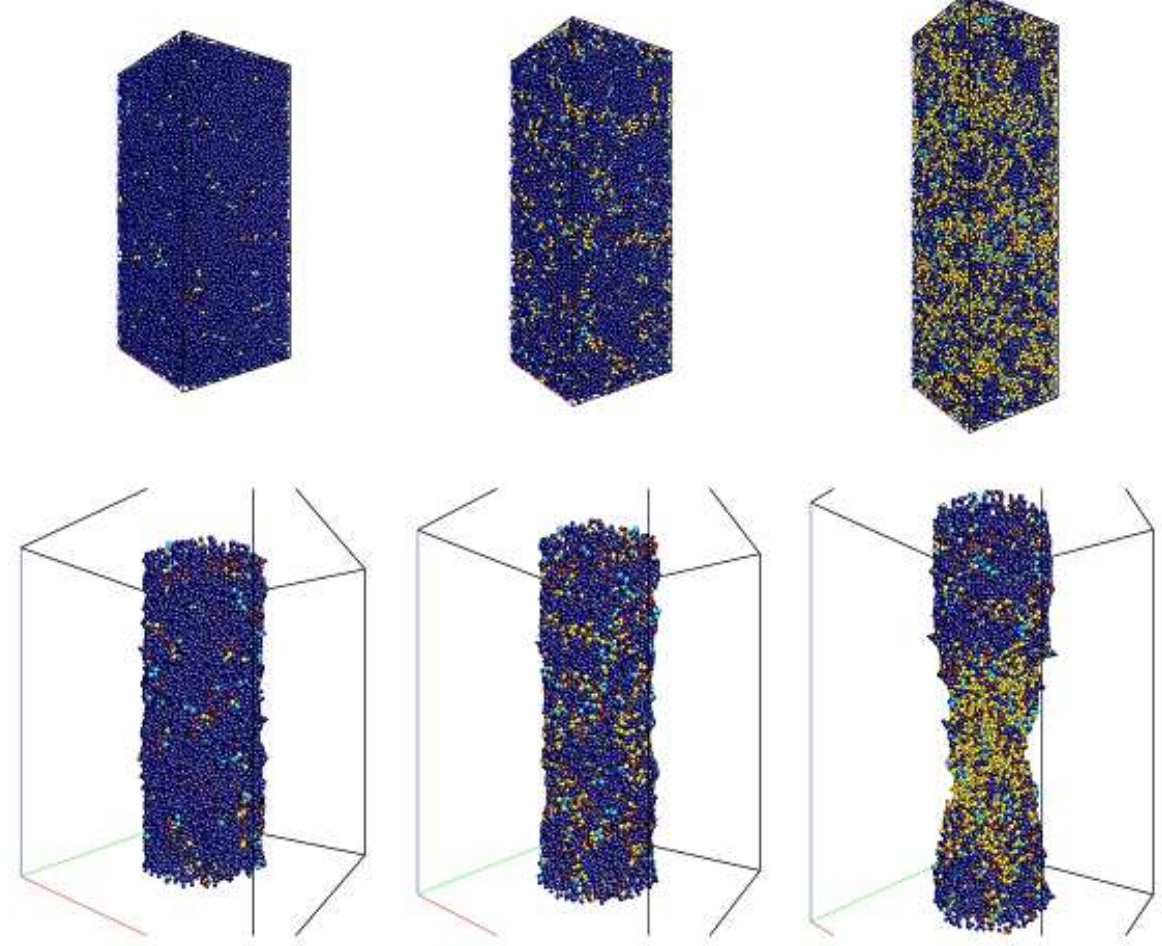

Figure S7 Snapshots of the simulations with the BKS Potential at strains of 10, 20, and $40 \%$. See Figure S5 for the color code of the atoms.

\section{Images about sample preparation}

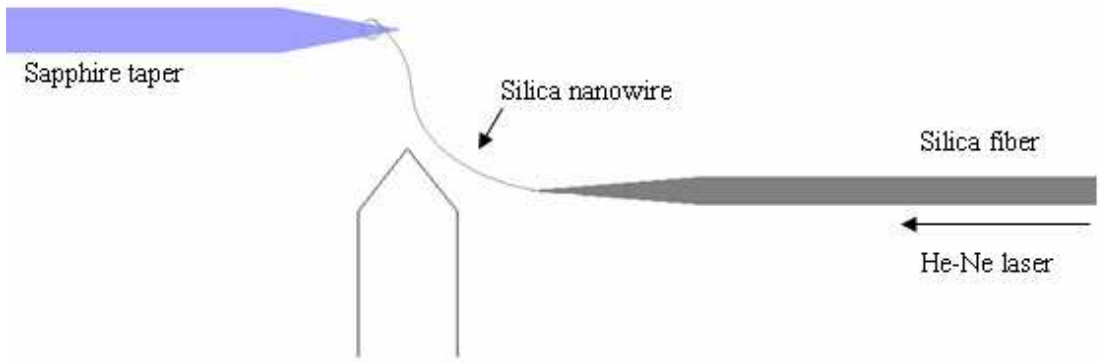

Flame

Figure S8 A schematic diagram illustrating a self-modulated taper-drawing process of ultrathin silica nanowires. 

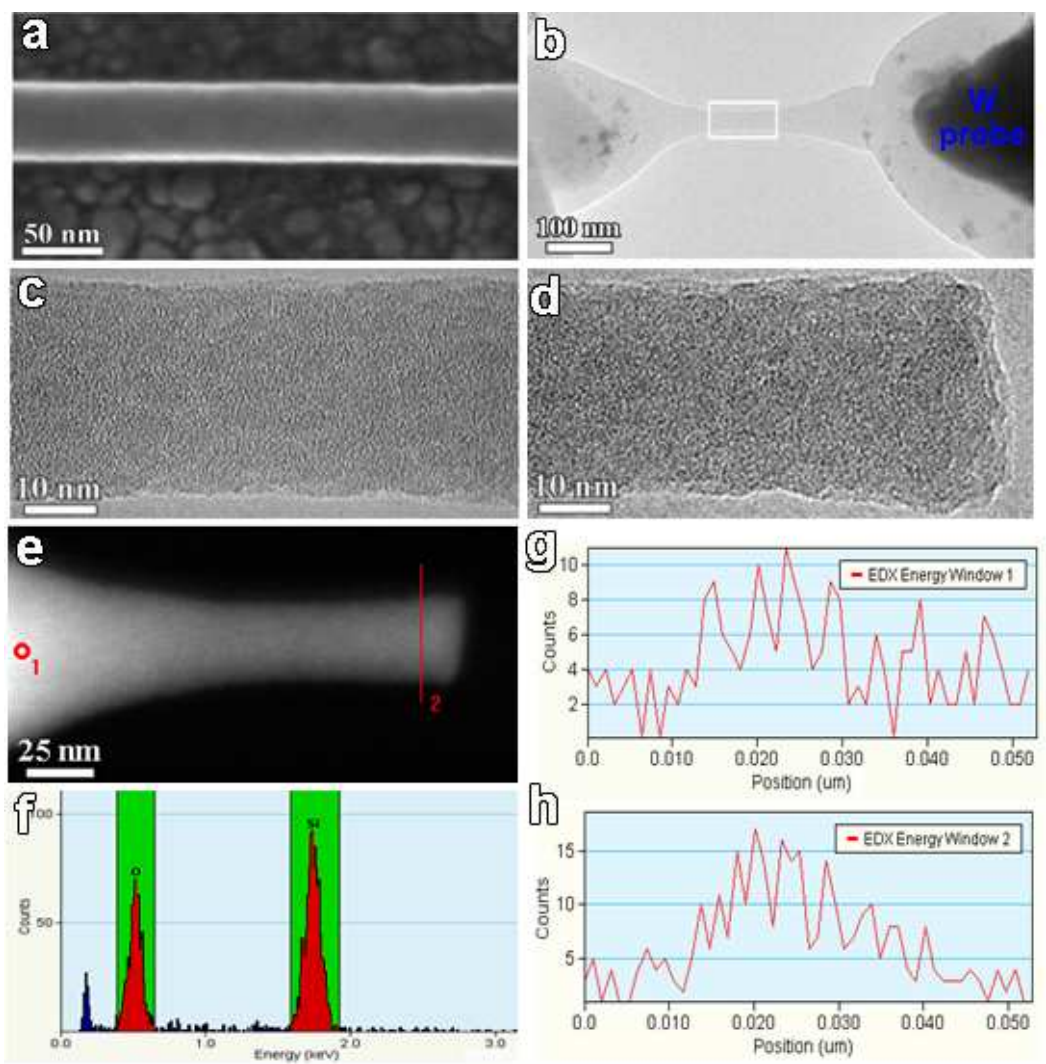

Figure S9 Structure and composition characterizations of the drawn dog-bone silica NWs. a, a SEM image of a $49.6 \mathrm{~nm}$ diameter nanowire; $\mathbf{b}$, a TEM image of a $28.2 \mathrm{~nm}$ diameter dog-bone sample; $\mathbf{c}$ was taken from the white framed region of $\mathbf{b}$; d, an HRTEM image of the broken part at the AFM tip side; e, an HAADF image of the broken part; $\mathbf{f}$, EDS analysis at position 1 of $\mathbf{e} ; \mathbf{g}$ and $\mathbf{h}$ are the EDX intensity profiles along a line marked by a " 2 " in e by selecting the EDS energy window of oxygen and silicon, respectively. 